\title{
Vault: Fast Bootstrapping for the Algorand Cryptocurrency
}

\author{
Derek Leung, Adam Suhl, Yossi Gilad, and Nickolai Zeldovich \\ MIT CSAIL
}

\begin{abstract}
Decentralized cryptocurrencies rely on participants to keep track of the state of the system in order to verify new transactions. As the number of users and transactions grows, this requirement becomes a significant burden, requiring users to download, verify, and store a large amount of data to participate.

Vault is a new cryptocurrency design based on Algorand that minimizes these storage and bootstrapping costs for participants. Vault's design is based on Algorand's proof-of-stake consensus protocol and uses several techniques to achieve its goals. First, Vault decouples the storage of recent transactions from the storage of account balances, which enables Vault to delete old account state. Second, Vault allows sharding state across participants in a way that preserves strong security guarantees. Finally, Vault introduces the notion of stamping certificates, which allow a new client to catch up securely and efficiently in a proofof-stake system without having to verify every single block.
\end{abstract}

Experiments with a prototype implementation of Vault's data structures show that Vault's design reduces the bandwidth cost of joining the network as a full client by $99.7 \%$ compared to Bitcoin and $90.5 \%$ compared to Ethereum when downloading a ledger containing 500 million transactions.

\section{INTRODUCTION}

Cryptocurrencies enable decentralized electronic payments, smart contracts, and other applications. However, supporting a large number of users and transactions will require cryptocurrencies to address two crucial and related bottlenecks: storage (how much data every participant needs to store) and bootstrapping (how much data every participant has to download to join the system). For example, in Bitcoin [21], a new client that wishes to join the network and verify that it received the correct state must download about $150 \mathrm{~GB}$ of data, as of January 2018 [3]. Storage and bootstrapping costs are related because, in a decentralized design, existing nodes must store enough state to help new nodes join the system.

Designing a cryptocurrency whose storage and bootstrapping costs scale well with the number of users and transactions is difficult due to several challenges. First, a cryptocurrency must prevent double-spending-that is, prevent a user from spending the same money twice or issuing the same transaction multiple times. This is typically done by keeping track of past transactions, but doing so is incompatible with good scalability. For instance, Bitcoin stores all past transactions, which does not scale well (costs grow linearly with the number of transactions). As another example, Ethereum [9] does not store all transactions but instead keeps track of the sequence

Network and Distributed Systems Security (NDSS) Symposium 2019 24-27 February 2019, San Diego, CA, USA ISBN 1-891562-55-X

https://dx.doi.org/10.14722/ndss.2019.23313

www.ndss-symposium.org number ("nonce") of the last transaction issued from a given account [28]. This nonce must be stored even if the account has no remaining balance. As a result, this does not scale well either (costs grow linearly with the number of old accounts) and has caused problems for Ethereum when a smart contract inadvertently created many zero-balance accounts [6], [29]. We measure the Ethereum ledger ( $\S$ VII) and find that $38 \%$ of Ethereum accounts have a balance of zero.

Second, a cryptocurrency relies on all participants to check the validity of transactions. This requires the participants to have enough state to validate those transactions. Storing all account balances allows a participant to validate any transaction but requires storage space that grows with the number of accounts. On the other hand, not storing all account balances could imply that fewer participants can vet transactions.

Third, proof-of-stake systems, such as Algorand [14], can provide high transaction throughput. However, such proof-ofstake systems are particularly challenging in terms of bootstrapping cost. Convincing a new participant of the validity of a block in the blockchain requires first convincing them of the balances (stakes) of all users in an earlier block. Convincing a new user of the validity of the latest block thus requires convincing them of the balances of all users at all points in time, starting with the initial genesis block.

Finally, an appealing way to reduce storage and bootstrapping costs is to delegate the job of storing state and certifying future states to a committee whose participants are trusted in aggregate. However, existing systems that take this approach [17], [18], [22] rely on long-standing committees known to an adversary. As a result, this adversary may be able to target the committee members, leading to security or availability attacks.

This paper presents Vault, a new cryptocurrency design based on Algorand that addresses the storage and bootstrapping bottlenecks described above. In particular, Vault reduces the bandwidth cost of joining the network as a full client by 99.7\% compared to Bitcoin and $90.5 \%$ compared to Ethereum when downloading a ledger containing 500 million transactions. Vault builds on Algorand's proof-of-stake consensus protocol and addresses the above challenges of storage and bootstrapping costs using several techniques:

First, Vault decouples the tracking of account balances from the tracking of double-spent transactions. Each Vault transaction is valid for a bounded window of time, expressed in terms of the position in the blockchain where the transaction can appear. This allows Vault nodes to keep track of just the transactions that appeared in recent blocks and to forget about all older transactions. The account balance state, on the other hand, is not directly tied to past transactions, and zero-balance accounts can be safely evicted. 
Second, Vault uses an adaptive sharding scheme that combines three properties: (1) it allows sharding the account state across nodes so that each node does not need to store the state of all accounts; (2) it allows all transactions to be validated by all nodes, using a Merkle tree to store the balance information; and (3) it adaptively caches upper layers of the Merkle tree so that the bandwidth cost of transferring Merkle proofs grows logarithmically with the number of accounts.

Finally, Vault introduces stamping certificates to reduce the cost of convincing new users of a block's validity. The insight lies in trading off the liveness parameter used in selecting a committee to construct the certificate of a new block [14], [22]. ${ }^{1}$ The stamping certificates are built on top of existing Algorand certificates and have a much lower probability of selecting an online quorum (so in many cases Vault fails to find enough participants to construct a valid certificate) but require fewer participants to form the certificate (thus significantly reducing their size) while still preserving the same safety guarantees (i.e., an adversary still has a negligible probability of corrupting the system). Building an extra layer of stamping certificates allows us to relax liveness for stamping without affecting the liveness of transaction confirmation. Vault's stamping certificates are generated in a way that allows new clients to skip over many blocks in one verification step.

We prototype and benchmark the core of Vault's design, focusing on bootstrapping and storage. Our evaluation shows that Vault's storage and bootstrapping cost is $477 \mathrm{MB}$ for 500 million transactions when account creation and churn rates match those observed in Ethereum in practice. This is a significant reduction compared to existing systems like Ethereum and Bitcoin; with the same 500 million transactions, Ethereum and Bitcoin would require $5 \mathrm{~GB}$ and $143 \mathrm{~GB}$ respectively. Individual microbenchmarks demonstrate that each of Vault's techniques are important in achieving its performance goals.

The contributions of this paper are:

- The design of Vault, a cryptocurrency that reduces storage and bootstrapping costs by $10.5-301 \times$ compared to Bitcoin and Ethereum and that allows sharding without weakening security guarantees.

- Techniques for reducing storage costs in a cryptocurrency, including the decoupling of account balances from doublespending detection and the adaptive sharding scheme.

- The stamping certificate technique for reducing bootstrapping costs in a proof-of-stake cryptocurrency.

- An evaluation of Vault's design that demonstrates its low storage and bootstrapping costs, as well as the importance of individual techniques.

\section{Motivation}

Vault's goal is to reduce the cost of storage and bootstrapping in a cryptocurrency. There are two significant aspects to this goal, corresponding to two broad classes of prior work.

The first is what we call the "width" of the ledger: how much data does each participant need to store in order to validate transactions (including detecting double-spending)? In

\footnotetext{
${ }^{1}$ Vault avoids the use of long-standing committees by using Algorand's cryptographic sortition and player-replaceable consensus.
}

Bitcoin, for example, the "width" is the set of all past unspent transactions [21]. Techniques that address the width of a ledger focus on managing the substantial storage costs of keeping the history of all transactions on each client. Vault reduces its "width" by decoupling account state from transaction state $(\S \mathrm{IV})$ and by adaptively sharding its state $(\S \mathrm{V})$.

The second is what we call the "length" of the ledger: how much data must be transmitted to a new participant as proof of the current state of the ledger? In Bitcoin's case, the proof consists of all block headers starting from the genesis block, chained together by hashes in the block headers, as well as all of the corresponding block contents (to prove which transactions have or have not been spent yet). Techniques addressing the length of the ledger typically allow clients to skip entries when verifying block headers, which reduces the total download cost. Vault reduces its "length" by using stamping certificates to omit intermediate state ( $\S \mathrm{VI})$.

Table I summarizes Vault's characteristics and compares them with other cryptocurrencies. Bitcoin and Ethereum [9] provide no formal guarantees on the correctness of the latest state. Permissioned cryptocurrencies have low bootstrapping cost but are vulnerable to an adversary which compromises a quorum of permissioned nodes at any point. A system combining OmniLedger [17] and Chainiac [22] lacks single points of failure, but even then an adversary may adaptively compromise a selected committee. Algorand [14] provides strong security guarantees, but its bootstrapping costs grow prohibitively quickly. Vault alone achieves cryptographic security against an adversary that can adaptively compromise users while scaling in both storage and bootstrapping costs. We explore related work in more detail in $\S$ VIII.

\section{OVERVIEW}

Vault is a permissionless, proof-of-stake cryptocurrency that significantly reduces new client bootstrapping costs relative to the state of the art by reducing both steady-state storage costs and the sizes of proofs needed to verify the latest state.

\section{A. Objectives}

Suppose Alice is a new participant in Vault who holds the correct genesis block. She wishes to catch up to the latest state and contacts Bob, an existing participant (or perhaps a set of participants). Vault should achieve the following main goals:

- Efficient Bootstrapping: If Bob is honest, he should be able to convince Alice that his state is correct and deliver this state using a minimal amount of bandwidth.

- Complete Bootstrapping: If Bob is honest, then upon synchronization, Alice has sufficient state to execute the entire protocol correctly. Moreover, Alice should now be able to help other new clients catch up.

- Safe Bootstrapping: If Bob is malicious, he should not be able to convince Alice that any forged state is correct.

- Efficient Storage: Bob must store a small amount of data to help Alice join the network.

Vault's design also confers additional benefits: 


\begin{tabular}{l|l|l|l} 
System & Execution State & Proof Size & Bootstrap Security \\
\hline Bitcoin [21] & UTXOs & Headers + TXs & Probabilistic (heaviest chain wins) \\
\hline Ethereum [9] & All accounts & Headers + All accounts & Probabilistic (heaviest chain wins) \\
\hline Permissioned & $\frac{\text { Live accounts }}{\text { Shards }}$ & Majority of trust set's signatures & Cryptographic if majority never compromised; none otherwise \\
\hline OmniLedger [17] + Chainiac [22] & $\frac{\text { UTXOs }}{\text { Shards }}$ & $\frac{\text { Headers+Certificates }}{\text { Sparseness }}+\frac{\text { UTXOs }}{\text { Shards }}$ & Cryptographic with static attacker; none with adaptive attacker \\
\hline Algorand [14] & UTXOs & Headers + Certificates + TXs & Cryptographic \\
\hline Vault & $\frac{\text { Live accounts }}{\text { Shards }}$ & $\frac{\text { Headers +Certificates }}{\text { Sparseness }}+\frac{\text { Live accounts }}{\text { Shards }}$ & Cryptographic
\end{tabular}

TABLE I. VAULT COMPARED TO OTHER CRYPTOCURRENCIES. UTXO REFERS TO UNSPENT TRANSACTION OUTPUTS; TX REFERS TO TRANSACTIONS.

- Charging for Storage: Adversaries must acquire significant stake to inflate the size of the protocol state.

- Liveness and Availability: Despite sharding state across clients, Vault continues to operate even when some users disconnect from the network. Additionally, Vault maintains bootstrap efficiency even when some users lose connectivity after a block is confirmed.

\section{B. Threat Model}

Vault should achieve its goals even in the face of adversarial conditions. However, many properties are unachievable given an arbitrarily strong attacker, which can indefinitely drop, delay, and reorder messages on a network [13]. We therefore limit the attacker's power with the following assumptions, inherited from Algorand [14]:

- Bounded Malicious Stake: At least some proportion $h$ of all money (the "stake") in Vault is controlled by honest users, where $h>\frac{2}{3}$. Stake sold by a user counts towards $h$ for some duration $d$ (e.g., 48 hours) following the sale.

- Cryptographic Security: The adversary has high but bounded computation power. In particular, the adversary cannot break standard cryptographic assumptions.

- Adaptive Corruptions: The adversary may corrupt a particular user at any time (given that at no point it controls more than $1-h$ of the stake in Vault).

- Weak Synchrony: The adversary may introduce network partitions lasting for a duration of at most $b$ (e.g., 24 hours). During a network partition, the adversary may arbitrarily reschedule or drop any message. The minimum time between network partitions is nontrivial (e.g., 4 hours).

\section{Algorand Background}

Vault's consensus protocol is based on Algorand, which we briefly review here. All users' clients in Vault agree on an ordered sequence of signed transactions, and this sequence constitutes the cryptocurrency ledger. Vault is a permissionless proof-of-stake system, meaning that any user's client, identified by a cryptographic public key, may join the system, and the client of any user who holds money may eventually be selected to append to the ledger. Honest clients listen for new transactions and append recent valid transactions to the ledger.

The frequency at which a user's client is selected is proportional to the user's stake. Sets of transactions are batched into blocks. Each block contains a block header, which contains a cryptographic commitment to the transaction set. Block headers also contain the cryptographic hash of the previous block in the ledger. Block headers are small, so these hashes allow clients to quickly verify historical transaction data.

Additionally, block headers contain a special pseudorandom selection seed $Q$. Before a client proposes a block, it computes $Q$ in secret, so $Q$ is unpredictable by the rest of the network and partially resistant to adversarial manipulation. As in Algorand, Vault uses $Q$ to seed Verifiable Random Functions (VRFs) [20] to implement cryptographic sortition. Cryptographic sortition produces a sample of the users in the system, weighted by the stake of their accounts. Each client's membership in the sample remains unknown to an adversary until the client emits a message because a VRF allows the client to compute this membership privately; since VRFs produce a proof of their correctness, any other client can verify this membership. To protect the system against adversaries which corrupt a user after that user is selected, clients sign their messages with ephemeral keys, which they delete before transmission.

Vault uses a Byzantine agreement scheme which operates in rounds. Each round, the protocol selects some block proposer which assembles the transaction set and header forming the block, which is broadcast via a peer-to-peer gossip network [12]. Subsequently, the protocol selects a committee which verifies the correctness of the block. To sample users in a manner resistant to adversarial manipulation, committees from round $r$ are seeded with the value of $Q$ from round $r-1$ and weighted by proofs of stake from round $r-b$.

Once clients become confident of a block's confirmation, Vault uses sortition to select a subset of clients to certify the block by signing its receipt (i.e., Algorand's "final" step). The aggregation of these signatures past some secure threshold, along with proofs of stake for each signature, forms a final certificate which proves to any client that a block is valid: the Byzantine agreement protocol guarantees that for each round, at most one valid block (or an empty block if the proposer misbehaves) reliably receives this certificate. Given knowledge of only the genesis (i.e., the first) block, a new client is convinced that the block from round $r$ is correct if a peer can produce $r-1$ block headers and the $r-1$ corresponding certificates of validity.

\section{System Design}

Figure 1 gives an overview of Vault's data structures, which are the key to Vault's lower storage and bootstrapping costs. The data structures are based around a chain of block headers, shown in the middle of the figure. Each block header consists of four elements: PREvBLOCK (the hash of the previous block), $Q$ (the seed for cryptographic sortition), TxRoot (a Merkle tree commitment [19] to the list of transactions in the 


\section{$\S \mathrm{VI}$}

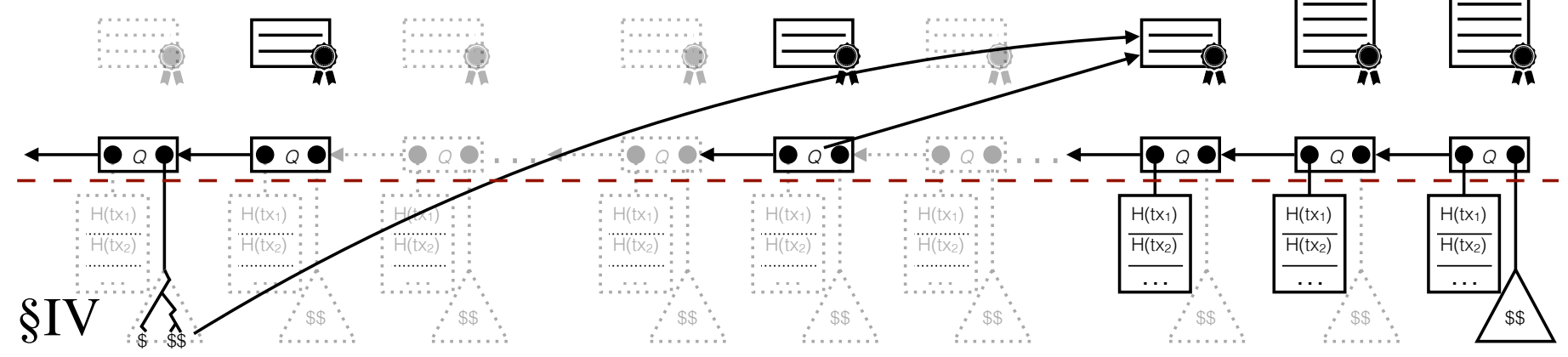

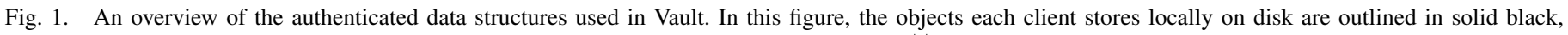

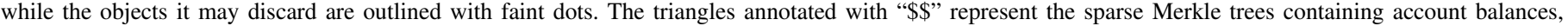

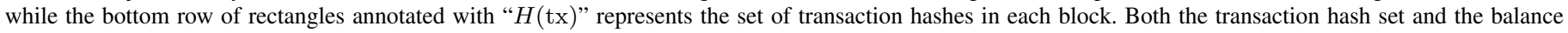

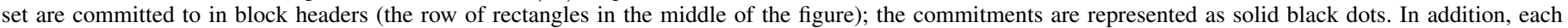

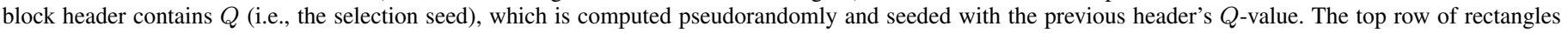

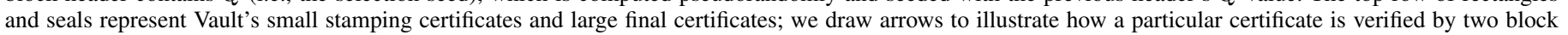
headers. Not shown is Vault's adaptive sharding $(\S \mathrm{V})$.

block), and BALRoOT (a sparse Merkle tree commitment [8] to the balances of every user after applying transactions).

Every block must follow four rules to be considered valid:

1) Transactions in the block are not expired. Each transaction includes the first and last block number (in the blockchain) where it can appear.

2) After all transactions in the block are executed, no account ends up with a negative balance.

3) Transactions in the block have not been executed before (i.e., have not appeared previously on the ledger).

4) BALROOT correctly reflects all users' balances after applying the block's transactions to the previous block's balances.

In order to check that a new block follows these rules, clients maintain two pieces of state, shown in solid black (as opposed to grayed out) in the bottom half of Figure 1:

- The tree of account balances from the most recent block. This allows a client to ensure that new transactions have sufficient funds (rule 2) and to verify the correctness of the new balance tree (rule 4).

- The lists of transactions from the last few blocks. This allows a client to ensure that a transaction has not appeared previously (rule 3) by checking that a new transaction does not appear in any of the previous transaction lists. To minimize the storage required by these lists, TXRoOT commits to a list of transaction hashes, rather than the transactions themselves.

Clients can discard transaction lists older than a certain threshold, corresponding to the maximum validity interval of a transaction, which we denote $T_{\max }$. Transactions that appeared more than $T_{\max }$ blocks ago will be rejected by rule 1 and need not be tracked explicitly.

$\S I V$ describes in more detail how clients check these rules while using a minimal amount of storage. $\S \mathrm{V}$ further describes Vault's adaptive sharding, which allows clients to store only a subset of the balance tree. These techniques combine to reduce the "width" of Vault's ledger.
Vault uses Algorand's consensus protocol to decide which valid block will be next in Vault's blockchain. The consensus protocol produces a final certificate confirming agreement on that block, shown in the top half of Figure 1. These certificates allow a new client to securely join the system and determine which chain of blocks is authentic.

Each certificate consists of a set of signatures (of the block header) by a committee of clients chosen pseudorandomly using cryptographic sortition. In order to verify a certificate, a new client must check that all of the signatures are valid (which is straightforward) and check that the clients whose signatures appear in the certificate were indeed members of the committee chosen by cryptographic sortition (which requires state). Verifying committee membership requires two pieces of state: the sortition seed $Q$, used to randomize the selection, and the balance tree at BALROOT, used to weigh clients by how much money their users have.

In Algorand's certificate for block $r$, BALROOT comes from block $r-b$, while $Q$ comes from block $r-1$. This means that, in order to verify block $r$, the client must first verify block $r-1$ so that the client knows the correct $Q$ for verifying block $r$ 's certificate. Furthermore, the committees used for Algorand's certificates are relatively large, so that with high probability there are enough committee members to form a certificate for each block. These certificates are shown with a tall rectangle at the top of Figure 1.

Vault introduces a second kind of certificate, called a stamping certificate, which speeds up bootstrapping. The stamping certificate differs from the final certificate in two important ways. First, instead of using $Q$ from the immediately previous block, it uses $Q$ from $b$ blocks ago. (For security, BALROOT must be chosen from $b$ blocks before $Q$, so this means BALROOT now comes from $2 b$ blocks ago.) This allows clients to "vault" forward by $b$ blocks at a time. Second, the stamping certificates use a smaller committee size, which makes the certificate smaller since it contains fewer signatures. The shorter rectangles at the top of Figure 1 represent stamping certificates, with arrows indicating the $Q$ and BALRoOT needed to verify them. 
Vault sets parameters so that the stamping certificate is just as hard for an adversary to forge as Algorand's original certificates. The trade-off, however, is that in some blocks, there may not be enough committee members to form a valid stamping certificate. To help new clients join the system, every Vault client keeps the stamping certificates for approximately every $b$ th block since the start of the blockchain, along with full Algorand-style certificates for the blocks since the last stamping certificate. Other certificates are discarded (shown as grayed out in Figure 1). $\S$ VI-B describes Vault's stamping certificates in more detail, which help Vault shrink the "length" of its ledger.

\section{EFFICIENT Double-SPENDING DETECTION}

This section describes Vault's design for minimizing the amount of storage required by a client to verify new transactions. To understand the challenges in doing so, consider the key problem faced by a cryptocurrency: double-spending. Suppose Alice possesses a single coin which she gives to both Bob and Charlie. A cryptocurrency must reject one of these transactions; if both are accepted, Alice double-spent her coin.

In Bitcoin, each transaction has a set of inputs and outputs. The inputs collect money from previous transactions' outputs, which can then be used by this transaction. The outputs define where the money goes (e.g., some may now be spendable by another user, and the rest remains with the same user). To detect double-spending in this scheme, Bitcoin must determine whether some output has been previously spent or not. Thus, clients must store the set of all unspent transaction outputs.

A more space-efficient approach is to store the balance associated with each user, rather than the set of unspent transactions. For example, Ethereum follows this approach. The cost savings from storing just the balances may be significant: for instance, there are ten times as many transactions in Bitcoin as there are addresses [2], [4].

Switching to a balance-based scheme introduces a subtle problem with transaction replay. If Alice sends money to Bob, Bob may attempt to re-execute the same transaction twice. In Bitcoin's design, this would be rejected because the transaction already spent its inputs. However, in a naïve design that tracked only account balances, this transaction still appears to be valid (as long as Alice still has money in her account), and Bob may be able to re-execute it many times to drain Alice's account.

To distinguish between otherwise identical transactions, Ethereum tags each account with a nonce, which acts as a sequence number. When an account issues a transaction, it tags the transaction with its current nonce, and when this transaction is processed, the account increments its nonce. The transactions issued by an account must have sequential nonces. Because of this design, Ethereum cannot delete accounts with zero balance; all clients must track the nonces of old accounts to prevent replay attacks, on the off chance that the account will receive money in the future.

Empty accounts significantly increase the storage cost of Ethereum. Our analysis of its ledger shows that approximately one-third of all Ethereum addresses have zero balance ( $\S \mathrm{VII})$. Worse, the inability to garbage-collect old accounts constitutes a serious denial-of-service vulnerability: an adversary with a

\begin{tabular}{|lr|}
\hline Alice $\rightarrow$ Bob: & $\$ 30$ \\
\hline Issuance: & 550 \\
Expiry: & 574 \\
\hline Nonce: & 8 \\
\hline & Mlice \\
\hline
\end{tabular}

Fig. 2. The format of a Vault transaction from Alice to Bob. In addition to the sender, receiver, and amount, the transaction contains $t_{\text {issuance }}, t_{\text {expiry }}$, and a nonce. A valid transaction contains the sender's digital signature.

small amount of money may excessively increase the cryptocurrency's storage footprint by creating many accounts. In fact, in 2016 an Ethereum user inadvertently created many empty accounts (due to a bug in Ethereum's smart contract processing) [6], requiring the Ethereum developers to issue a hard fork to clean up the ledger [29]. ${ }^{2}$

At a high level, Vault avoids the problem of storing empty accounts by forcing transactions to expire. The rest of this section describes Vault's solution in more detail.

\section{A. Transaction Expiration}

All transactions in Vault contain the fields $t_{\text {issuance }}$ and $t_{\text {expiry, }}$ which are round numbers delineating the validity of a transaction: blocks older than $t_{\text {issuance }}$ or newer than $t_{\text {expiry }}$ may not contain the transaction. Moreover, we require that $0 \leq$ $t_{\text {expiry }}-t_{\text {issuance }} \leq T_{\max }$ for some constant $T_{\max }$. This way, a verifying client may detect the replaying of a transaction by checking for its presence in the last $T_{\max }$ blocks. (Transactions still contain a nonce to distinguish between otherwise identical transactions; however, this nonce is ephemeral and not stored.) As a result, clients do not need to track account nonces and can delete empty accounts from the balance tree. Figure 2 shows the format of one transaction.

Requiring transaction lifetimes to be finite means that, if a transaction fails to enter a block before it expires (e.g., because its transaction fee was lower than the current clearing rate), the issuer must reissue the transaction in order for the transaction to be executed. On the other hand, the expiration time ensures that old transactions that failed to enter a block when they were originally issued cannot be re-entered into a block at a much later time by an adversary; i.e., expiration bounds the outstanding liabilities of an account.

Transactions are valid for a window of rounds and not just a single round because clients must account for uncertainty in transaction propagation latencies, transaction fees, and round durations. For example, by the time a transaction propagates through the network, some number of rounds will have passed since the issuer signed it. If the transaction were valid for just one round, the issuer must precisely estimate its propagation latency. Similarly, a temporary spike in the transaction fees could occur due to a burst of high demand. A window of transaction validity amortizes over these uncertainties.

The choice of $T_{\max }$ affects two considerations. The first is that clients must store the last $T_{\max }$ blocks' worth of

\footnotetext{
${ }^{2}$ Currently, Ethereum transaction fees are high enough to make such attacks unlikely. However, proposed cryptocurrency designs like Algorand [14] aim to support orders of magnitude more throughput, which would lead to lower transaction fees, and which would in turn make such attacks worth considering.
} 
transactions to detect duplicates; a larger $T_{\max }$ increases client storage. (Clients can store transaction hashes instead of the transactions themselves to reduce this cost.) The second is that clients must reissue any transaction that fails to enter a block within $T_{\max }$ (if they still want to issue that transaction). In our experiments, we set $T_{\max }$ to the expected number of blocks in 4 hours (which, based on Algorand's throughput of $\sim 750 \mathrm{MB} /$ hour [14], suggests at most a few hundred megabytes of recent transaction hashes); we believe this strikes a balance between the two constraints.

Although Vault's design requires that transactions be valid for a short window of time (e.g., 4 hours), Vault is nonetheless compatible with applications that require transactions to be settled far in the future. For instance, payment channels are used to collapse multiple off-chain transactions into a single on-chain settlement transaction. By aggregating many off-chain transactions over a long period of time, payment channels can reduce on-chain transaction load. In Vault, a settlement transaction can be postdated into future rounds-e.g., a window of rounds that will appear in a week. Thus, even though the transaction itself is valid for a relatively short window of $T_{\max }$, the payment channel can still aggregate off-chain transactions for an arbitrary time period (e.g., an entire week).

\section{B. Efficient Balance Commitments}

To efficiently commit to the large set of balances in BALRoOT, Vault clients build Merkle trees [19] over this set. Each leaf in the Merkle tree stores a single account-that is, the public key of the account and the balance for that account. The leaves are sorted by public key. With a Merkle tree, clients may prove that some object exists in a given set using a witness of size $\mathcal{O}(\log n)$, where $n$ is the total number of objects in the set. This allows them to efficiently construct proofs of stake. For example, for a balance set containing 100 million accounts (4 GB of on-disk storage), it suffices for a client to send $1 \mathrm{~KB}$ of data to prove its stake against a 32-byte BALROOT in a block header. It is important for the proofs of stake in Vault to be small since a certificate may contain thousands of these proofs (see $\S \mathrm{VI})$.

For clients to verify the validity of BALROOT for a new block, BALROOT must be deterministically constructed given a set of balances. As a result, the Merkle leaves are sorted before they are hashed together to create the root. Since a leaf may be deleted when an account balance reaches 0 , Vault uses sparse Merkle trees [8] to perform balance commitments. A sparse Merkle tree possesses the structure of a prefix trie, which allows us to perform tree insertions and deletions with a Merkle path witness of size $\mathcal{O}(\log n)$. In fact, a witness of size $\mathcal{O}(\log n)$ is sufficient for a client to securely update BALROOT without storing the corresponding Merkle tree. We exploit this self-verifying property of Merkle witnesses in $\S \mathrm{V}$.

\section{Safe and Complete Bootstrapping}

With these two mechanisms, it suffices to bootstrap a new verifier by transmitting a commitment to the latest state and the last $T_{\max }$ rounds of transactions.

This scheme is complete because it allows a new verifier to detect double-spending. Suppose the current round is $r$, and a transaction was confirmed in round $r_{0}$. Reading the set of transactions in the last $T_{\max }$ rounds is sufficient to detect that this transaction is a duplicate. Either $r-T_{\max } \leq r \leq r_{0}$, so it is in this set and therefore invalid, or $r_{0}<r-T_{\max }$, which means the transaction must have expired at $r_{0}+T_{\max }<r$.

Moreover, this scheme is safe due to the cryptographic integrity property of Merkle trees. For an attacker to forge a cryptocurrency state, it must tamper with the preimage to a Merkle tree, which is computationally reducible to the security of a cryptographic hash function.

\section{Sharding Balance Storage}

As the number of accounts in Vault grows, the cost of storing balances becomes the primary bottleneck. Concretely, each account requires about 40 bytes (32 bytes for a public key and 8 bytes for the balance). This means that if there were 100 million accounts, every Vault client would need to store about $4 \mathrm{~GB}$ of data, which may be acceptable (e.g., it is less than Bitcoin's current storage cost). On the other hand, if Vault grew to $10 \times$ or $100 \times$ more accounts, the storage cost would likely be too high for many clients.

To address this problem, Vault shards the tree of balances across clients, pseudorandomly distributed by the client user's key. Sharding allows clients to deal with large ledger sizes. As an extreme example, consider a system with 100 billion accounts and 1 million online clients. Setting the number of shards to 1,000 would require each client to store approximately $4 \mathrm{~GB}$ of data (i.e., 1/1000th of the balances), rather than the full 4 TB balance tree. Even with a large number of shards in this example, every shard's data is held by about 1000 clients, ensuring a high degree of availability.

One challenge in sharding is that fewer clients now have the necessary data to verify any given new transaction. Existing proposals (like OmniLedger [17]) implement sharding by restricting verifiers' responsibility of preventing double-spending attacks to their own shards. These proposals seem attractive because they reduce not just storage costs but also bandwidth and latency costs, allowing the system to scale throughput arbitrarily. Unfortunately, such schemes are vulnerable to adversaries which control a fraction of the currency. As shard size decreases, so do shards' replication factors, and as a result, transactions in a given shard are verified by a small number of clients. An adversary may own a critical fraction of the stake in a shard by chance, giving it control of the entire shard. Thus, these systems require an undesirable trade-off between scaling and security, which in practice limits the degree of sharding.

Vault's design allows sharding without any reduction in security because all clients retain the ability to verify all transactions. The trade-off comes in terms of increased bandwidth costs during normal operation (which may reduce the system's maximum throughput): as we describe in the rest of this section, with Vault's adaptive sharding, each transaction must include a partial Merkle proof, which grows proportionally to the height of the balance tree.

\section{A. Shard Witnesses}

Vault shards the tree of account balances into $2^{N}$ pieces by assigning an account to a shard according to the top $N$ bits of the account's public key. A client stores a shard by storing 
the balances for the accounts in that shard. Clients store the shard(s) corresponding to their user's public key(s). Clients that join the network, or create a new account, download the corresponding shards from existing peers.

The first challenge is to support sharding without a loss in security by ensuring that all clients can verify all transactions. Recall that the balance set is stored in a sparse Merkle tree ( $\mathrm{IV}-\mathrm{B})$ whose root is committed to in the block header. These trees support insertions, updates, and deletions with witnesses of size $\mathcal{O}(\log n)$. To allow a client to check the validity of any transaction in a proposed block (even if that transaction operates on accounts outside of this client's shard), Vault transactions include Merkle witnesses for the source and destination accounts in the previous block's BALROOT. Any client that possesses the previous block's BALROOT can use the Merkle witnesses to confirm the source and destination account balances and thus to verify the transaction.

\section{B. Updating Witnesses}

The second challenge is that the structure of the Merkle tree storing account balances might change from the time the transaction is issued to the time the transaction is committed to the ledger, invalidating the transaction's witnesses. Vault clients maintain a pool ("mempool" in Bitcoin terminology) of pending transactions that have not yet been committed. Suppose a client has transaction $T_{0}$ in its pool, and in the meantime, a new block is committed to the ledger, containing transaction $T_{1}$ but not $T_{0}$. Applying $T_{1}$ to the Merkle tree causes the tree's internal nodes to be updated (since each node is a hash of its children), and as a result, the witnesses for transaction $T_{0}$ are no longer valid. A naïve implementation of Vault would thus be vulnerable to denial-of-service attacks: an adversary can send a tiny amount of money to a victim's account, which invalidates pending transactions issued by the victim, and prevents the victim from spending money.

To address this challenge, Vault leverages the fact that witnesses for a transaction need not be signed along with the rest of the transaction. Since the witnesses are self-certifying, the witnesses can be updated without requiring the issuer to re-sign the entire transaction. Building on this insight, Vault clients update the witnesses of pending transactions when any concurrent transactions are applied to the ledger.

Specifically, suppose that a client has an existing witness for account $A$ (which might be the source or destination account associated with $T_{0}$ ), and makes a concurrent change to account $B$ (which might be associated with $T_{1}$ ). Each witness is a path from a leaf node in the Merkle tree (e.g., accounts $A$ or $B$ ) to the root of the Merkle tree. For any two witnesses, there must be an intersection point (the lowest node in the tree where the two witnesses overlap). To update the existing witness for account $A$ after a change to account $B$, Vault recomputes $B$ 's witness with the new leaf value for $B$, then finds the intersection between $A$ 's and $B$ 's witnesses, and finally splices the top part of $B$ 's new witness into $A$ 's witness. This produces a fresh witness for $A$ that is valid after $B$ is modified.

The witness update algorithm described above can be executed by any client, even if the client does not store the shards associated with any of the transactions. It relies only on the fact that the newly committed transactions have fresh

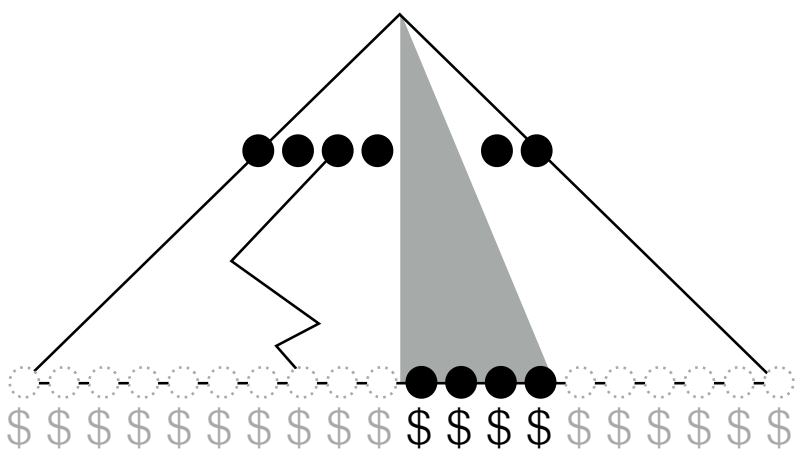

Fig. 3. An illustration of a single Vault shard and the balance Merkle tree. Dots here represent Merkle nodes, and the "\$" symbols represent account balances. The solid black dots and dark "\$" symbols represent the balances which are part of the shard (the shaded gray triangle), while those in gray represent the parts of the tree which are not. The row of black dots in the middle represent the frontier of Merkle nodes stored by all clients regardless of shard assignment. The jagged line connecting one of these nodes to an unstored leaf represents the Merkle witness necessary for updating a balance.

witnesses, which is ensured by the client that proposed this block of transactions in the first place.

\section{Adaptive Sharding: Truncating Witnesses}

The third challenge is that witnesses increase transaction size. For example, if the transaction size is 250 bytes (on par with Bitcoin), and there are 100 billion accounts in the system, a single Merkle witness will hold 37 sibling nodes in expectation, which is $1.2 \mathrm{~KB}$. Two witnesses would introduce $2.4 \mathrm{~KB}$ of overhead per transaction-almost an $11 \times$ increase. Note that the inclusion of Merkle witnesses increases bandwidth but not storage costs: since all blocks are certified by an honest committee, verifiers discard the witnesses after they recompute BALROOT. Thus, the trade-off applies only to the bandwidth costs of broadcasting transactions during rounds.

To manage the overhead of larger witnesses, clients store (in addition to their shards) an intermediate frontier that cuts across the Merkle tree-roughly speaking, the subset of tree nodes at some depth. Storing this frontier allows clients to verify partial witnesses, which prove the path from a leaf node to the frontier, rather than all the way to BALRoOT. Figure 3 illustrates one such partial witness.

We can quantify the trade-off between transaction size and the cost of storing the frontier. Moving the frontier up in the tree by one level (i.e., going from the nodes in the frontier to their parents) increases the length of a partial Merkle witness by a single sibling. Moving the frontier up in the tree by one level also halves its size. Vault can thus tune the trade-off between the size of partial Merkle witnesses in each transaction and the amount of storage required for the frontier.

If the frontier lies in the dense region of the Merkle prefix tree (towards the top of the tree), the shape of the frontier is simple: it involves all the Merkle nodes at a given level. However, if the frontier lies near the leaves of the Merkle prefix tree (near the bottom), a client cannot simply store all the nodes at a given level, as the layers are larger than the balance set (owing to the sparseness of the Merkle tree). Instead, these frontiers assume a "jagged" shape; they are defined as the nodes which sit at a fixed height from the bottom of the tree. 
To update a node in the frontier, it suffices for a client to observe a witness and follow these two rules: (1) if the witness increases the height of a frontier node, the client replaces that frontier node with its children (which were present in the witness); and (2) if the witness decreases the height of a frontier node, the client replaces that frontier node with its parent (if it did not previously store the parent). Note that the length of the witness alone is sufficient for determining whether an insertion, an update, or a deletion occurred.

By application of the coupon collector's problem ${ }^{3}$, we see that if there are approximately $n \log n$ account balances, then in expectation the last dense layer is of depth $n$ [5]. For example, if there are 100 billion $\approx 2^{37}$ accounts, then layer $n=32$ is the last dense one.

\section{Safe and Complete Bootstrapping}

Vault's adaptive sharding construction maintains the cryptographic integrity of Merkle trees, as any verifier can construct the Merkle proof of any account's stake on demand by concatenating the prefix of the proof, which the verifier stores, with the suffix of the proof, which is provided by the spender and dynamically updated by the verifier. Therefore, this construction is safe and complete.

\section{Succinct Ledger CertificAtes}

Bootstrapping a new client in a proof-of-stake system like Algorand requires transferring a significant amount of data to the new client, due to two factors. First, the confirmation of each block depends on the state of the system at the time the block was proposed. For instance, as mentioned in $\S I I I-C$, the Algorand committee that forms the final certificate of a block is selected based on the random seed $Q$ from the previous block. Thus, to verify the correctness of block $r$, a new client must first verify the correctness of block $r-1$ in order to obtain the correct $Q$ value for verifying block $r$. Second, in Algorand's design, the certificate confirming a block consists of a large number of signatures, reflecting the large committee size. This arrangement is shown at the top of Figure 4.

Vault addresses this problem using two techniques. First, Vault introduces a stamping certificate that can be verified using state from $b$ and $2 b$ blocks ago rather than the state from 1 block ago. This allows clients to "leapfrog" by $b$ blocks at a time instead of having to verify every single block in the blockchain. Vault uses Algorand's cryptographic sortition to privately select a committee for stamping certificates in a way that does not reveal committee membership to an adversary in advance. This ensures that an adversary cannot selectively corrupt members of this committee to falsify a certificate. Certificate signatures use the ephemeral keys of each committee member; a committee member destroys its keys before it broadcast its signature. This is shown in the middle of Figure 4 and described in more detail in $\S \mathrm{VI}-\mathrm{A}$.

Second, Vault uses a smaller committee size to generate the stamping certificates, which reduces the size of the certificates

\footnotetext{
3 The coupon collector's problem can be stated as follows: suppose there are $\mathrm{n}$ distinct types of coupons. A drawn coupon's type is chosen uniformly at random. How many draws are required to collect one coupon of each type?
}

themselves (since they contain fewer signatures). ${ }^{4}$ To ensure that a smaller committee does not give the adversary a higher probability of corrupting the committee, Vault requires a much larger fraction of expected committee members to vote in order for the stamping certificate to be valid. This means that, with significant probability, the committee fails to gather enough votes to form a stamping certificate. However, this is acceptable because new clients have two fallback options: they can either verify Algorand's full certificate, or they can verify a stamping certificate for a later block and backtrack using PREVBLOCK hashes in the block headers. This arrangement is shown at the bottom of Figure 4 and described in $\S \mathrm{VI}-\mathrm{B}$.

\section{A. Leapfrog Protocol}

To enable leapfrogging, Vault constructs a sortition committee for the stamping certificate of block $r$ using the seed $Q$ from block $r-c$, where $c \geq 1$ is some constant. For security, the proof-of-stake balances must be selected from $b$ blocks before the seed $Q$, so they are chosen from block $r-c-b$. Members of this committee wait for consensus on block $r$, and once consensus is reached, they broadcast signatures for that block (after deleting the corresponding ephemeral signing key), along with proofs of their committee selection. The set of these signatures forms the stamping certificate for BlockHeader ${ }_{r}$.

As mentioned above, this committee is, in principle, known as soon as block $r-c$ has been agreed upon. However, the committee is selected in private using cryptographic sortition, and honest clients do not reveal their committee membership until they vote for block $r$, which prevents an adversary from adaptively compromising these committee members.

Now each certificate depends on two previous block head-

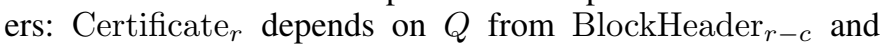
BALRoOT from BlockHeader ${ }_{r-c-b}$. Moreover, Certificate ${ }_{r}$ validates BlockHeader $r$, which itself contains the value of $Q$ used for Certificate ${ }_{r+c}$ and the value of BALROOT used for Certificate $_{r+c+b}$.

To optimize for a new client catching up on a long sequence of blocks starting with the genesis block, we set $c=b$, so that the client does not need to validate separate blocks for $Q$ s and BALRoOTs. This reduces the bootstrapping bandwidth by a factor of $b$, since a new client needs to download and authenticate every $b$ th block header and certificate.

To ensure that any client can help a new peer bootstrap, all clients store the block header and certificate for blocks at positions that are a multiple of $b$. Additionally, to ensure that the base case is true, the first $2 b$ blocks in Vault are predetermined to be empty. Finally, to quickly catch up after momentarily disconnecting from the network, clients keep the previous $2 b$ block headers at all times.

Choosing $b$. Vault's choice of $b$ trades off the weak synchrony assumption (i.e., partitions last for periods shorter than $b$ ) against $d$, the speed at which sold stake becomes malicious. We briefly justify our choice of $b$ below; we refer the reader to Algorand's security analysis [15] for a formal treatment.

\footnotetext{
${ }^{4}$ Note that multi-signatures [1] would not significantly reduce the size of the certificates since the certificate needs to include a proof of cryptographic sortition (VRF) and a partial Merkle proof for each committee member whose signature appears in the certificate, which cannot be aggregated away.
} 


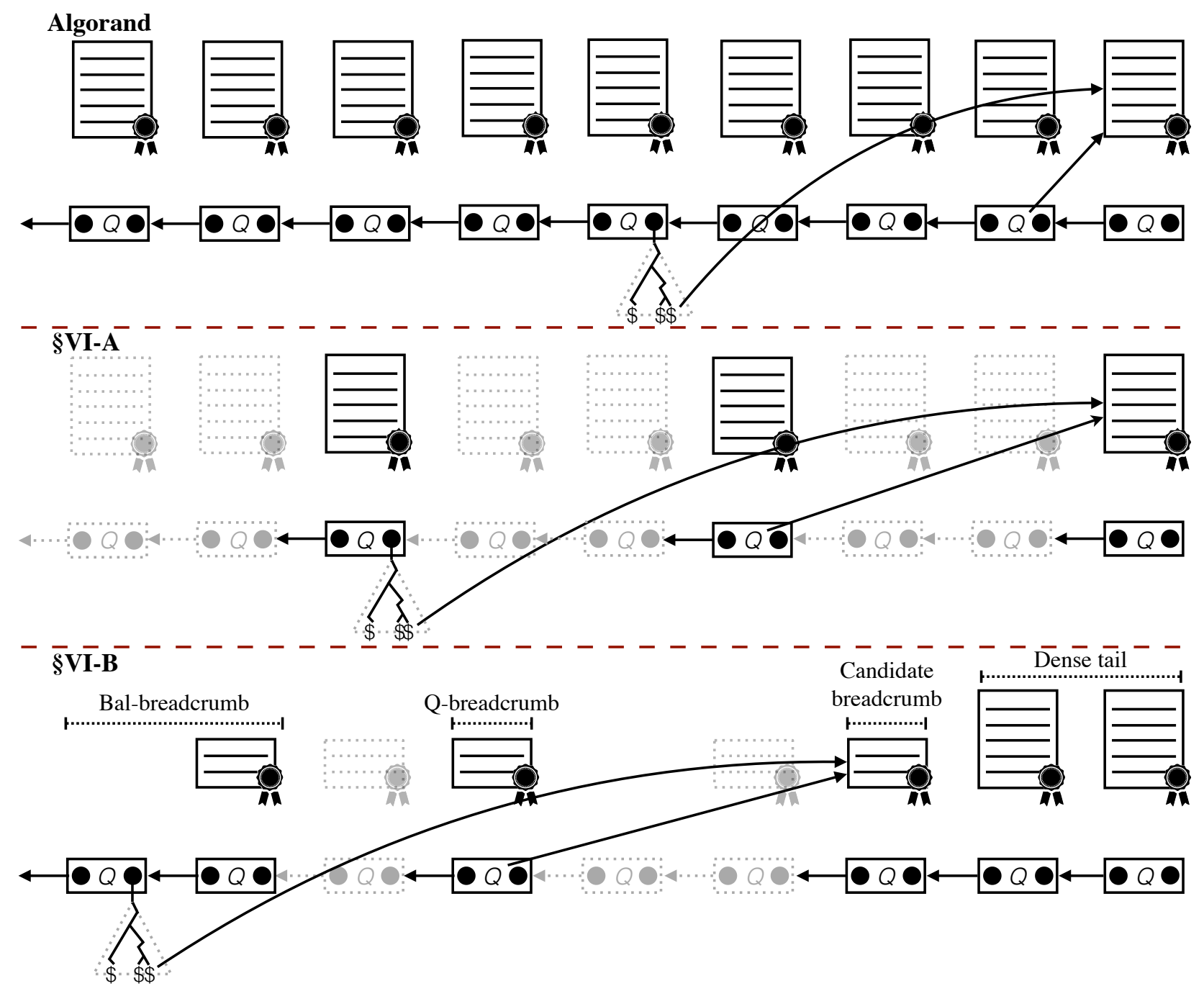

Fig. 4. Two optimizations used to reduce the bandwidth needed to prove validity of the latest state. In this figure, $b=c=3$; as before, objects that clients can discard are outlined with dots. The top figure depicts the basic ledger data structure without any optimization: a large final certificate authenticates each block header, and each certificate depends on the $Q$ value immediately before it and the proofs of stake $b$ blocks ago. The next figure shows the additional stamping certificate chain with the leapfrog optimization: each leapfrogging certificate depends on the value of $Q$ from $b$ blocks ago and the balances from $2 b$ blocks ago ( $(\mathrm{VI}-\mathrm{A})$. The bottom figure shows stamping committee optimization used to reduce the size of certificates ( $\S \mathrm{VI}-\mathrm{B})$. It illustrates the candidate, Q-, and Bal-breadcrumbs which consist of a small stamping certificate and block header along with a tail of block headers. It shows the dense tail formed by the large final certificates that prove validity of the current block. (The figure omits the frozen breadcrumbs, which are farther back in the chain.)

On the one hand, suppose the adversary partitions the network for more than $b$ blocks starting at round $r^{\prime}$. Then the adversary may predict the value of $Q$ at round $r^{\prime}+b$ and then manipulate its public keys at round $r^{\prime}$ such that it controls both the proposer and the committee at round $r^{\prime}+b+1$. In this way, the adversary confirms two different blocks and thus creates a fork at round $r^{\prime}+b+1$. Therefore, $b$ must be large enough to resist complete partitions.

On the other hand, suppose a rich, honest user sells off $50 \%$ of the stake in Vault at round $r^{\prime}$. A few rounds after the user completes the sale, a poor adversary corrupts this user, who by chance controls a supermajority of the committee at round $r^{\prime}+b+1$. Then again the adversary gains control of the ledger. Although this adversary controls little of the system's current stake, it controls much of the system's past stake. As a result, $b$ must be small enough to allow honest users to finish participating in Vault after selling off their stake.
Since $c$ introduces an extra delay to certificate creation, for security we require that not $b \leq d$ but instead $b+c \leq d$, and since we set $c=b$ we require that $2 b \leq d$. At Vault's highest level of throughput, $2 b=d=2880$ corresponds to about two days' worth of blocks.

\section{B. Stamping Committees}

Algorand's consensus protocol requires thousands or tens of thousands of signatures to produce a final certificate for a block. This threshold is very high because Algorand guarantees a very low rate of failure in terms of liveness and safety. A failure in liveness prevents a block from being confirmed, while a failure in safety may produce a ledger fork.

As with final certificates, a stamping committee threshold should be set sufficiently high such that an adversary cannot gather the signatures required to trick a new client into accepting a forged ledger fork with high probability. Since 
adversaries know when they are selected for a leadership in advance, and a certificate must be secure for all time, we must keep a strict safety threshold.

Although we cannot relax safety, we can greatly relax liveness. Suppose a new client has already verified the block headers for blocks $r$ and $r+b$, using stamping certificates, but there was no stamping certificate produced for block $r+2 b$ due to relaxed liveness requirements. If there was a stamping certificate produced for block $r+2 b-1$, the new client can efficiently verify that stamping certificate and block instead.

Specifically, the new client can ask an existing peer for the headers of blocks $r-1$ and $r+b-1$ and efficiently verify them by checking PREVBLOCK hashes in blocks $r$ and $r+b$ respectively. Since headers are relatively small, this costs the client little bandwidth. We use the term breadcrumb to denote this chain of PREVBLOCK pointers from a stamping certificate to an earlier block header. Figure 4's bottom row shows two breadcrumbs: one that required backtracking by one block (for BALROOT), and one that required no backtracking (for $Q$ ).

If the stamping certificate at $r+2 b-1$ also failed to form, Vault repeats this process to find the latest block before $r+2 b$ that did have a stamping certificate. If no such block exists in a $b$-block interval, Vault falls back to a full Algorand certificate.

Given that stamping certificate creation may occasionally fail, each breadcrumb must contain a small "tail" of block headers which are required to certify the two subsequent breadcrumbs produced at most $b$ and $2 b$ blocks ahead, respectively. Since block headers are relatively small (less than 256 bytes), the cost of storage here is low (less than 1.3 MB for $b=1440)$. As clients observe the confirmation of new blocks and the successful creation of new stamping certificates, they update their state so as to minimize the sizes of these tails. Clients must also hold a dense tail of block headers and final certificates at the end of the ledger for each block after the last header for which a stamping certificate was produced. Vault clients discard this dense tail whenever new stamping certificates are successfully created.

\section{Safe and Complete Bootstrapping}

Ledger certificates completely commit to the state of the ledger by signing a Merkle commitment to this state ( $\S \mathrm{IV}-\mathrm{B}$ ). Therefore, it suffices to show that this state is the correct one.

While $Q$ is usually unpredictable and random, an adversary may introduce bias into its value during network partitions. Given this bias, Vault requires a safety failure rate of $2^{-100}$ for both its final and stamping certificates. However, with a relaxed liveness assumption, we can decrease stamping certificate size by at least an order of magnitude.

For example, with an honesty rate of $h=80 \%$, a final certificate requires a threshold of 7,400 signatures. If we allow stamping certificates to fail to form $65 \%$ of the time, then it suffices to have a threshold of 100 signatures (out of a suitably smaller committee). Applying the stamping optimizations allows clients in Vault to verify the latest block header in a 10year old ledger by downloading $365 \mathrm{MB}$ or less. Appendix $\S \mathrm{A}$ analyzes stamping certificate size given other settings of the honesty and liveness failure rates.

\section{Storage Requirements}

We next describe the objects that clients keep to allow convincing a new client that the ledger state is valid, and we describe the invariants that apply to each of these components. An algorithm for keeping these objects up-to-date follows.

Proof-objects stored. The bottom of Figure 4 illustrates the objects that a Vault client needs to store.

- The dense tail is the set of all headers and full final certificates since the candidate breadcrumb.

- The candidate breadcrumb is the breadcrumb with the lastobserved stamping certificate. The candidate breadcrumb is tentative and may be overwritten by a "better" breadcrumb (i.e., a more recent breadcrumb which makes the candidate breadcrumb obsolete). This breadcrumb is never more than $b$ blocks ahead of the Q-breadcrumb.

- The $Q$-breadcrumb is the breadcrumb with the stamping certificate immediately preceding the candidate breadcrumb. This breadcrumb's certificate has been fixed as no subsequent certificate may be better than this. However, its tail of block headers may not yet be trimmed.

- The Bal-breadcrumb is the breadcrumb with the stamping certificate immediately preceding the Q-breadcrumb. Like the Q-breadcrumb, its certificate is final and unchanging. Moreover, its tail remains "minimal" as new certificates are seen. In other words, it maintains the shortest tail such that the following conditions are true:

1) It contains the block header needed to authenticate the Q-breadcrumb's certificate's $Q$-value.

2) It contains the block header needed to authenticate the candidate breadcrumb's certificate's proofs of stake.

- Frozen breadcrumbs are all of the earlier breadcrumbs, from the start of the blockchain to the Bal-breadcrumb. As the blockchain grows (adds new blocks with their respective certificates), breadcrumbs "graduate" from being a candidate breadcrumb, to a Q-breadcrumb, to a Bal-breadcrumb, and eventually to the set of frozen breadcrumbs. It is these frozen breadcrumbs that allow a new client to quickly catch up from the initial genesis block to recent blocks.

Keeping proof-objects up-to-date. All clients maintain a proof of the ledger's latest block. A client mutates its proof on observing two events from the cryptocurrency network:

- When a client observes a block and a full final certificate, it appends them to its dense tail.

- When a client observes a new stamping certificate later than its candidate breadcrumb, it deletes the final certificate of the "stamped" round and all older final certificates. Moreover, it moves ownership of the corresponding block headers to the new stamping certificate, which becomes the "new" breadcrumb. Next,

- If the new breadcrumb's round is not more than $b$ blocks greater than the Q-breadcrumb's, the client replaces its candidate breadcrumb with the new breadcrumb, transferring ownership of the block headers.

o Otherwise, the client: 
1) Freezes the Bal-breadcrumb, adding it to the set of frozen breadcrumbs.

2) Sets the Q-breadcrumb as the new Balbreadcrumb. This breadcrumb's tail becomes trimmable.

3) Sets the candidate breadcrumb as the new Qbreadcrumb. This breadcrumb's position is now optimal and fixed (assuming that stamping certificates are received in order).

4) Sets the new breadcrumb as the candidate breadcrumb.

Finally, it "trims" the tail of the Bal-breadcrumb to keep its length minimal.

\section{Evaluation}

The primary question that our evaluation answers is, "How effective is Vault at reducing the bandwidth cost of helping a new client join the network?" $§ V I I-B$ presents the results.

To understand why Vault achieves a reduction in bandwidth, we further answer three questions targeted at each of Vault's techniques, as follows. Recall that two components contribute to bootstrapping costs: the state needed to execute the protocol and the bandwidth required to convince a new client that this state is correct.

- Balance Pruning: How much does transaction expiration reduce storage cost by? (§VII-C)

- Stamping Certificates: What are the cost savings of using Vault's sparse sequence of stamping certificates for bootstrapping? ( $(\mathrm{VII}-\mathrm{D})$

- Balance Sharding: What are the trade-offs involved in sharding Vault's balance sets? ( $(\mathrm{VII}-\mathrm{E})$

\section{A. Experimental Setup}

To answer the questions above, we implemented the data structures needed to execute the Bitcoin, Ethereum, Algorand, and Vault protocols. However, we have not integrated these data structures into their respective systems; in particular, we have not evaluated real-time characteristics of Vault, such as its transaction latency or throughput. We vary transaction volumes between 50 and 500 million transactions, and we fill all blocks with the maximum number of transactions given a fixed block size. (As of February 2018, there are around 300 million transactions in Bitcoin [4] and around 150 million transactions in Ethereum [10].) We ignore the storage cost of auxiliary data structures required to efficiently update a protocol's state; for example, we do not implement database indexes.

Algorand uses a transaction format similar to Bitcoin's. We consider only simple transactions with the form of one input and two outputs (one to the receiver and the other to self).

The ratio of unique accounts to transactions on Ethereum is around 15\% [11], [10] as of January 1, 2018. Additionally, we obtained the Ethereum ledger up to this date by synchronizing a Parity [23], [24] Ethereum client (in fatdb mode). Our analysis of the Ethereum state indicates that around 38\% of all accounts have no funds and no storage/contract data (i.e., only an address and a nonce). For Ethereum and Vault, we fix the account creation rate at $15 \%$ and the churn rate at $38 \%$.

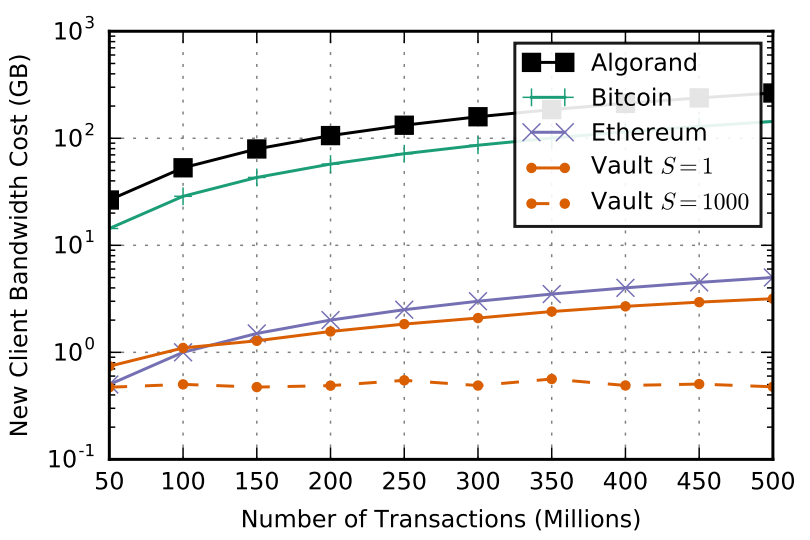

Fig. 5. An end-to-end comparison of cryptocurrency bootstrapping costs Compared to Bitcoin and Algorand, Vault and Ethereum reduce storage costs by one to two orders of magnitude. Vault outperforms Ethereum at 150 million transactions because it can delete old accounts. Sharding Vault with a factor of 1,000 reduces the costs of storing balances to a negligible amount, and the total storage cost remains low (below $500 \mathrm{MB}$ ) even with 500 million transactions on the ledger. Note that the $y$-axis is logarithmic.

Other than to count the number of empty accounts, we do not consider the costs in Ethereum which result from per-account data storage or from smart contracts.

We instantiate the following parameters both in Algorand and in Vault:

- $80 \%$ of the stake in the system is honest $(h=0.8)$.

- Stake sold off by a later-corrupted user counts towards $h$ for $d=48$ hours.

- Network partitions last for at most 2 days. (Recall that during a network partition, an adversary may arbitrarily reschedule and drop any message.) This implies that the leapfrogging interval is $b=1440$ rounds ( $\S \mathrm{VI}-\mathrm{A}$ ).

- The maximum transaction lifetime is $T_{\max }=4$ hours. This keeps the cost of storing the hashes of recent transactions to the hundreds of megabytes.

- Stamping certificates fail to form at a rate of $65 \%$. This implies that a certificate contains $T_{\text {stamping }}=100$ signatures, and a stamping sortition produces $\tau_{\text {stamping }}=120$ committee members in expectation $(\S \mathrm{A})$.

- The size of a block is $10 \mathrm{MB}$. (Lower block sizes are possible; these reduce both throughput and latency.)

We use $S$ in the rest of this section to denote the number of shards in Vault.

\section{B. End-to-End Evaluation}

Figure 5 presents the results of an end-to-end evaluation of Bitcoin, Ethereum, Algorand, and Vault (with sharding factors of $S=1$ and $S=1000$ ).

Algorand's storage cost exceeds that of Bitcoin. Every transaction that Bitcoin stores must also be stored by Algorand. In addition, supporting secure bootstrapping in Algorand incurs an additional cost ranging from 4 to $47 \mathrm{~GB}$, growing linearly with the number of confirmed transactions in the system.

Figure 5 shows clear gains in storing the set of account balances rather than the set of transactions. Vault and Ethereum, 


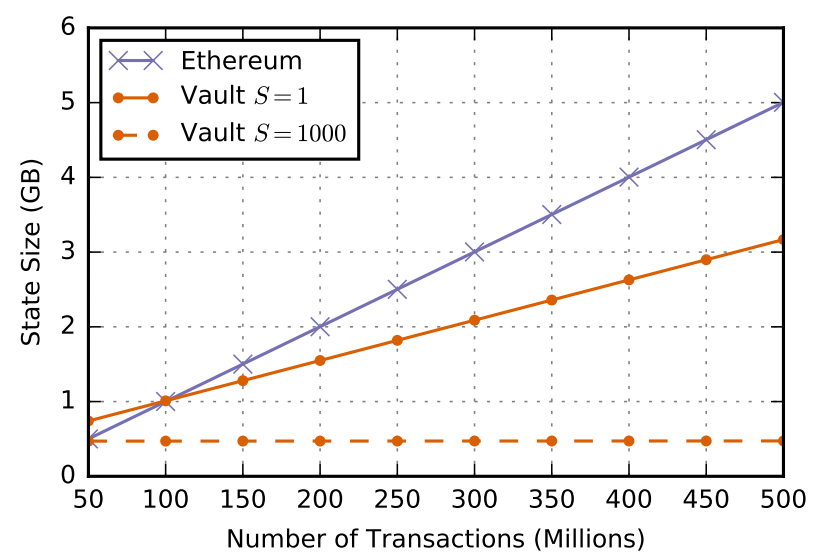

Fig. 6. A comparison of steady-state storage costs between Vault and Ethereum, given an account churn rate of $38 \%$. Pruning empty balances allows Vault to store less data than Ethereum past around 100 million transactions, even as Vault must store its recent transaction log. Note that the line associated with $S=1000$ grows linearly but appears flat due to its low slope.

which both store account balances, outperform Algorand and Bitcoin by 1 to 2 orders of magnitude. This holds both because the set of balances is much smaller than the set of all transactions, and also because an individual balance entry is smaller than a transaction itself. Given that we only consider simple transactions with one input and two outputs, we expect more complex transactions to amplify this effect.

Moreover, at 150 million transactions, Vault outperforms Ethereum even without sharding. This follows from the fact that Vault may delete accounts with no balance, which reduces overall storage cost by about $38 \%$. However, before 150 million transactions, the fixed cost of storing the recent transactions outweighs these savings. We note that throttling the throughput of Vault or reducing $T_{\max }$ decreases this cost.

Finally, sharding Vault reduces storage even more significantly. However, sharding is no "free lunch"; it increases the sizes of transactions and thus the steady-state bandwidth cost of propagating them to the entire network ( $\S \mathrm{VII}-\mathrm{E}$ ).

\section{Balance Pruning}

To evaluate the efficiency of Vault's balance pruning technique, we compare the storage footprint of Vault's balance set (again sharded at factors of 1 and 1000) against Ethereum's. Since Vault also requires a log of the recent transaction history to detect double-spending, we include these costs as well.

Figure 6 shows that the ability to prune the balance tree significantly reduces the ledger's storage costs at scale. Initially, Vault clients must hold the past 9.6 million transaction hashes to enforce transaction expiration, which costs around $307 \mathrm{MB}$ of overhead (if transaction expiration $T_{\max }$ is set to correspond to 4 hours). However, past 150 million transactions, holding the set of account balances dominates the cost of detecting doublespending. Since Ethereum clients cannot garbage collect the $38 \%$ of empty accounts in their balance trees, they must store these accounts in perpetuity. ${ }^{5}$ Maintaining a $\log$ of

\footnotetext{
5 We speculate that the use of Ethereum's smart contracts to programmatically create temporary accounts only exacerbates this problem. Efficient garbage collection implies cheap temporary account creation.
}

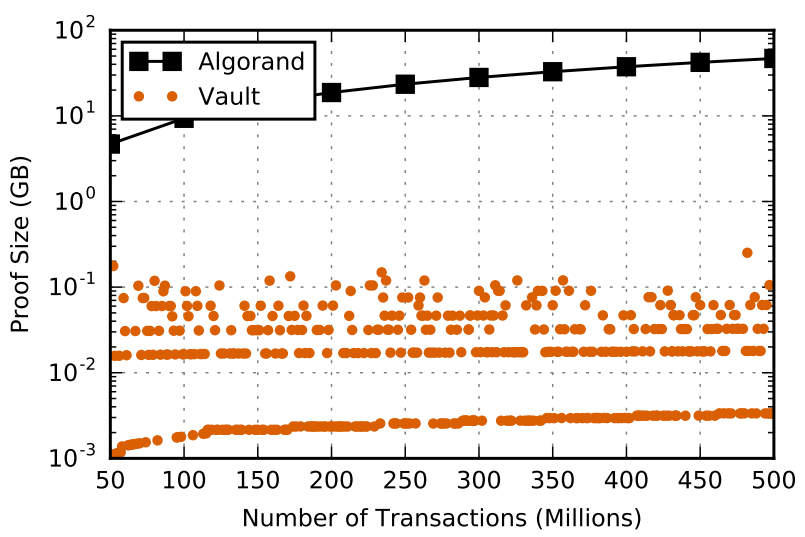

Fig. 7. A comparison between the certificate chain sizes in both Vault and Algorand. The proof size is $2-4$ orders of magnitude smaller in Vault, and its size does not exceed $1 \mathrm{~GB}$ up to 500 million transactions. Usually, the size of this proof remains under $100 \mathrm{MB}$. In the plot, proof sizes cluster around several bands, which correspond to the number of final certificates present in the dense tail. The lowest band grows linearly with the number of stamping certificates that were formed. Note that the y-axis is logarithmic.

recent transactions constitutes a constant storage cost, while the overhead of storing empty accounts grows linearly as the system continually processes new transactions.

\section{Stamping Certificates}

Next, we evaluate how efficiently a client can prove the validity of its state to a new peer. We measure the amount of data transferred for the certificate chain in Vault and compare it against the data transferred for the certificate chain in Algorand. Since the creation of stamping certificates in Vault is non-deterministic, we evaluate the amount of data transferred using fine-grained steps for the independent variable (number of transactions processed) to capture these effects.

Figure 7 reveals that the overhead of the certificate and header storage cost becomes significant in Algorand. To catch up to a ledger with 500 million transactions, a client must download around $47 \mathrm{~GB}$ of data.

In contrast, Vault's proofs are much smaller even though the use of a balance-based ledger increases the size of certificates (by including partial Merkle proofs); these proofs are almost always less than $100 \mathrm{MB}$ in size. Two factors decrease the size of these proofs. First, the chain of certificates is much sparser. On average, downloading an extra stamping certificate allows a client to validate an additional $b=1440$ blocks. Second, each individual stamping certificate is small. Instead of 7,400 signatures, each certificate consists of 100 signatures.

This evaluation demonstrates that, without stamping certificates, certificates would dominate the data required for bootstrapping. A $3.4 \mathrm{~GB}$ state size for balances matters little if $47 \mathrm{~GB}$ is necessary to prove its validity. Reducing the proof overhead to less than $100 \mathrm{MB}$ allows Vault to securely bootstrap new clients with modest bandwidth cost.

\section{E. Balance Sharding}

Under sharding, we would like to determine how decreasing the overhead of storing the intermediate frontier in the balance tree $(\S \mathrm{V}-\mathrm{C})$ increases the size of transactions. We fix 


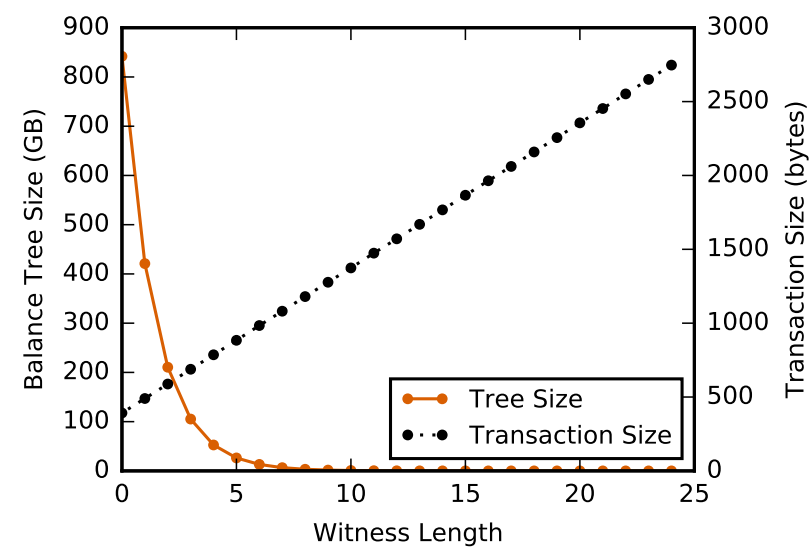

Fig. 8. Cost of storing sharded account balances and transaction sizes given some setting of the witness length. The tree stores 10 billion accounts, divided into $S=1000$ shards. Increasing transaction size linearly (i.e. extending the Merkle witness) decreases storage overhead exponentially.

the number of accounts to be 10 billion and the number of shards to be $S=1000$. Figure 8 illustrates this interaction.

We see that shard size is not the limiting factor. With $S=1000$, each client stores only 10 million accounts per shard, which costs less than $500 \mathrm{MB}$ each. Instead, sharding costs are dwarfed by the overhead of keeping the internal Merkle nodes on the frontier, which allow clients to verify transactions in other shards without needing to receive the entire Merkle path as part of the transaction. On the one hand, all clients may simply store all leaf Merkle nodes, which adds nothing to transaction overhead but also reduces storage cost by only a small amount: storing the set of balances along with the Merkle frontier costs each client almost 1 TB. On the other hand, the exponential fanout of the sparse Merkle tree provides diminishing returns on storing each subsequent layer; extending the Merkle witness by one hash halves the storage footprint of the Merkle nodes. Eventually, storage costs converge to the size of a shard.

\section{RELATED WORK}

In this section we contrast Vault with related systems and discuss their compatibility with Vault's techniques.

\section{A. Steady-State Savings: The "Width" Approach}

Several cryptocurrencies adopt mechanisms which reduce the amount of bandwidth needed to join the protocol (as a verifier) and the amount of storage needed to run the protocol.

Ethereum [9] summarizes account balances and other state into a short digest using Patricia Merkle Trees [16]. Each block commits to the root of the current tree, which allows new clients to obtain balance state from any untrusted node and then verify this state against a known Merkle root. To prevent an attacker from replaying a transaction issued by a user, users embed a sequence number (a nonce) in each transaction. Ethereum clients must track the last nonce issued by each account in the balance tree, even if the account is empty; otherwise, an old transaction could be replayed (e.g., if an empty account receives a deposit in the future). As we note in $\S \mathrm{IV}$, this means that Ethereum's storage cost grows with the number of all accounts that ever existed, leaving Ethereum vulnerable to denial-of-service attacks that create many temporary accounts. By decoupling account balances from tracking double-spent transactions ( $(\mathrm{IV})$, Vault prevents storage costs from growing with the number of old accounts. We believe that Vault's decoupling can be adopted by Ethereum to avoid unbounded storage for old accounts: by mandating expiration dates in all transactions, Ethereum can allow nodes to delete old state.

OmniLedger [17] shards its ledger by users' public keys, running Byzantine agreement on many ledgers in parallel. OmniLedger performs load balancing across each shard to improve throughput and reduce bandwidth and storage costs proportionally to the number of shards. Sharding allows OmniLedger to scale horizontally. However, OmniLedger requires a long-standing committee to run the PBFT [7] protocol to establish consensus on the ledger's state; this leaves it vulnerable to a strong adversary which may quickly corrupt validators. Moreover, its shard size and thus scalability is sensitive to the proportion of malicious users. Unfortunately, adapting OmniLedger to use Algorand in place of PBFT is non-trivial: it is unclear how Algorand's distinctly sampled committees, which persist not for an epoch but rather change with each message, can exchange state across shards without excessive bandwidth cost. Vault's adaptive sharding $(\S \mathrm{V})$ reduces the storage cost per participant and remains secure against an adversary that can quickly corrupt users, but its throughput does not increase with sharding.

An alternative approach to reducing the ledger's "width" is to record fewer transactions on the ledger. The Lightning Network [26] establishes payment channels between pairs of users. Participants in the channel post collateral on the ledger and then exchange transactions off the ledger to record their debts. As a result, by posting only two transactions on the ledger, a pair of participants may process arbitrarily many offledger transactions in a payment channel as long as it contains sufficient collateral to absorb them. One advantage of this scheme is that participants do not need to broadcast transactions that take place within a payment channel. We discuss how payment channels can be implemented on top of Vault in $\S I V-A$. However, payment channels come with limitations: they support only pairwise payment relationships, the collateral posted by each participant limits channel capacity, and their incentives assume that participants always act to maximize their payout. A key benefit of Vault is that its storage costs scale with the total number of accounts, rather than the number of transactions, which allows for more transactions to take place "on chain" without the limitations of payment channels.

MimbleWimble [25] uses an accumulator-like signature sinking scheme to "compact" blocks together according to the amount of work proved in the block header. Combined blocks eliminate transaction outputs which have been spent, reducing the state a verifier is required to download. Switching to a balance-based scheme like Vault's may allow MimbleWimble to increase its compaction savings by committing not to the set of unspent transactions but rather the current set of balances.

Observe that Vault can safely prune old balances by forcing transactions to expire quickly ( $(\mathrm{IV})$, and Vault can shard balances over the nodes in the network for a decrease in storage cost $(\S \mathrm{V})$. These schemes work independently of Algorand's 
specific characteristics (including proof of stake). Any cryptocurrency which maps cryptographic identifiers to balances and commits to them in a Merkle tree can transparently leverage these techniques.

\section{B. Short Proofs of State: The "Length" Approach}

Other cryptocurrencies observe that a small block header is often sufficient evidence of a block's validity. Therefore, they reduce the cost of verifying the block header sequence by shortening it. This allows clients to efficiently prove the validity of their state at any particular point in time.

Like Vault's stamping certificates, Chainiac's [22] Collective Signing (CoSi) [27] scheme allows a committee of verifiers to jointly produce a proof that a particular block is correct. As in Vault, verifying committees for some block also certify the correctness of blocks into the future; upon observing a block confirmation, committees produce forward links to the block. Since these links are arranged in a skiplist-like configuration, they allow verifiers to quickly bootstrap to the current state. However, Chainiac's scheme is inherently vulnerable to an adversary that can adaptively corrupt users because its committees are not secret. Sometime after the protocol designates a committee, an adversary which compromises this committee can forge a proof that a false view of the ledger is valid and thus deceive new clients into accepting a bogus state. Since the committees that produce Vault's certificate signatures are secretly selected and emit exactly one message, Vault's certificates resist attacks from adversaries that can adaptively corrupt clients. Chainiac may thus replace its committees with Vault's committees to improve its bootstrapping security.

MimbleWimble also reduces the length of the ledger. Blocks with more work supersede prior blocks with less work; since an adversary must possess significant processing power to attack these blocks, the proof of work requirements increase the new verifiers' confidence in these blocks. As in Bitcoin, this approach does not produce a proof of blocks' correctness, since an adversary that controls the network can prevent a user from ever observing the block with the largest amount of work. Vault builds on Algorand for reaching consensus, which ensures safety (no forks) with cryptographic guarantees even in the presence of network partitions.

We observe that in a permissioned cryptocurrency, where a supermajority of ledger writers are trusted, a signed checkpoint suffices to convince a new verifier that the state is correct [7]. Stellar [18] can be thought of in similar terms, where a core node will accept a checkpoint from nodes in its quorum set. Vault targets a permissionless setting where users do not configure trusted sets of known writers or trusted core nodes. As a result, Vault authenticates checkpoint signatures using cryptographic sortition, based on techniques from Algorand.

In general, permissionless cryptocurrencies where randomly sampled committees execute a consensus algorithm (e.g., Chainiac) can use Vault's stamping certificates transparently ( $\S \mathrm{VI})$. Simply embed the stamping certificates' seed $Q$ (or an existing randomness source) into blocks, and use a Sybil-resistant mechanism (e.g., weigh members via proof of work/stake) to select stamping committees.

\section{CONCLUSION}

Vault is a new cryptocurrency design based on Algorand that reduces storage and bootstrapping costs. Vault achieves its goals using three techniques: (1) transaction expiration, which helps Vault decouple storage of account balances from recent transactions and thus delete old account state; (2) adaptive sharding, which allows Vault to securely distribute the storage of account balances across participants; and (3) stamping certificates, which allow new clients to avoid verifying every block header, and which reduce the size of the certificate. Experiments demonstrate that Vault reduces the storage and bootstrapping cost for 500 million transactions to $477 \mathrm{MB}$, compared to $5 \mathrm{~GB}$ for Ethereum and $143 \mathrm{~GB}$ for Bitcoin.

\section{ACKNOWLEDGMENTS}

We thank the anonymous reviewers and our shepherd, Aniket Kate, for helping us improve this paper. We also thank Georgios Vlachos for assisting us with our security analysis of the certificates. Additionally, we thank David Lazar, Anish Athalye, and the rest of the MIT PDOS group for providing feedback on drafts of this paper. This work was supported by NSF awards CNS-1413920 and CNS-1414119.

\section{REFERENCES}

[1] M. Bellare and G. Neven, "Multi-signatures in the plain public-key model and a general forking lemma," in Proceedings of the 13th ACM Conference on Computer and Communications Security (CCS), Alexandria, VA, Oct.-Nov. 2006, pp. 390-399.

[2] BitInfoCharts, "Bitcoin rich list," https://bitinfocharts.com/ top-100-richest-bitcoin-addresses.html, 2018.

[3] Blockchain Luxembourg S.A., "Blockchain size," https://blockchain. info/charts/blocks-size, 2018.

[4] —, "Total number of transactions," https://blockchain.info/charts/ n-transactions-total, 2018.

[5] G. Blom, L. Holst, and D. Sandell, Problems and Snapshots from the World of Probability. Springer Science \& Business Media, 2012.

[6] V. Buterin, "Security alert [11/24/2016]: Consensus bug in geth v1.4.19 and v1.5.2," https://blog.ethereum.org/2016/11/25/ security-alert-11242016-consensus-bug-geth-v1-4-19-v1-5-2/, 2016.

[7] M. Castro and B. Liskov, "Practical Byzantine fault tolerance and proactive recovery," ACM Transactions on Computer Systems, vol. 20, no. 4, Nov. 2002.

[8] R. Dahlberg, T. Pulls, and R. Peeters, "Efficient sparse Merkle trees: Caching strategies and secure (non-)membership proofs," in Proceedings of the 21st Nordic Conference on Secure IT Systems, Nov. 2016, pp. 199-215.

[9] Ethereum Foundation, "Ethereum," 2016, https://www.ethereum.org/.

[10] Etherscan, "Ethereum transaction chart," https://etherscan.io/chart/tx, 2018.

[11] _ _ "Ethereum unique address growth rate," https://etherscan.io/chart/ address, 2018.

[12] K. R. Fall and W. R. Stevens, TCP/IP Illustrated, Volume 1: The Protocols. Addison-Wesley, 2011.

[13] M. J. Fischer, N. A. Lynch, and M. S. Paterson, "Impossibility of distributed consensus with one faulty process," in Proceedings of the 2nd ACM SIGACT-SIGMOD Symposium on Principles of Database Systems (PODS), Atlanta, GA, Mar. 1983, pp. 1-7.

[14] Y. Gilad, R. Hemo, S. Micali, G. Vlachos, and N. Zeldovich, "Algorand: Scaling Byzantine agreements for cryptocurrencies," in Proceedings of the 26th ACM Symposium on Operating Systems Principles (SOSP), Shanghai, China, Oct. 2017, pp. 51-68.

[15] _ "Algorand: Scaling Byzantine agreements for cryptocurrencies," Cryptology ePrint Archive, Report 2017/454, May 2017, http://eprint. iacr.org/. 
[16] D. E. Knuth, The art of computer programming. Pearson Education, 1997, vol. 3.

[17] E. Kokoris-Kogias, P. Jovanovic, L. Gasser, N. Gailly, and B. Ford, "OmniLedger: A secure, scale-out, decentralized ledger," Cryptology ePrint Archive, Report 2017/406, Feb. 2018, http://eprint.iacr.org/.

[18] D. Mazières, "The Stellar consensus protocol: A federated model for internet-level consensus," https://www.stellar.org/papers/ stellar-consensus-protocol.pdf, 2014.

[19] R. C. Merkle, "A digital signature based on a conventional encryption function," in Proceedings of the 7th Annual International Cryptology Conference (CRYPTO), Santa Barbara, CA, Aug. 1987, pp. 369-378.

[20] S. Micali, M. O. Rabin, and S. P. Vadhan, "Verifiable random functions," in Proceedings of the 40th Annual IEEE Symposium on Foundations of Computer Science (FOCS), New York, NY, Oct. 1999.

[21] S. Nakamoto, "Bitcoin: A peer-to-peer electronic cash system," https: //bitcoin.org/bitcoin.pdf, 2008.

[22] K. Nikitin, E. Kokoris-Kogias, P. Jovanovic, N. Gailly, L. Gasser, I. Khoffi, J. Cappos, and B. Ford, "Chainiac: Proactive software-update transparency via collectively signed skipchains and verified builds," in Proceedings of the 26th USENIX Security Symposium, Vancouver, Canada, Aug. 2017, pp. 1271-1287.

[23] Parity Technologies, "Parity," https://www.parity.io/, 2017.

[24] —, "Github - paritytech/parity: Fast, light, robust Ethereum implementation," https://github.com/paritytech/parity, 2018.

[25] A. Poelstra, "Mimblewimble," Oct. 2016, https://download.wpsoftware. net/bitcoin/wizardry/mimblewimble.pdf.

[26] J. Poon and T. Dryja, "The Bitcoin Lightning network: Scalable off-chain instant payments," Jan. 2016, https://lightning.network/ lightning-network-paper.pdf.

[27] E. Syta, I. Tamas, D. Visher, D. I. Wolinsky, P. Jovanovic, L. Gasser, N. Gailly, I. Khoffi, and B. Ford, "Keeping authorities "honest or bust" with decentralized witness cosigning," in Proceedings of the 37th IEEE Symposium on Security and Privacy, San Jose, CA, May 2016, pp. $526-545$.

[28] G. Wood, "Ethereum: A secure decentralised generalised transaction ledger," Ethereum Project Yellow Paper, vol. 151, pp. 1-32, 2014.

[29] _- "State trie clearing (invariant-preserving alternative)," https:// github.com/ethereum/EIPs/blob/master/EIPS/eip-161.md, 2016.

\section{APPENDIX}

\section{A. Stamping Certificate Security Analysis}

Recall that the security of a certificate is equivalent to the security of the committee that produced it. We first define two desirable properties of Vault's stamping certificates:

Definition. A certificate has a safety failure rate of $f_{s}$ if over all committees produced by cryptographic sortition, the probability that an adversary can obtain two distinct and validating certificates for a given block is $f_{s}$.

Definition. A certificate has a liveness failure rate of $f_{l}$ if over all committees produced by cryptographic sortition, the probability that the honest users fail to produce a certificate for a given block is $f_{l}$.

In these committees, an honest verifier releases its signature after it sees a block confirmation. Confirmed blocks are forksafe, so if one honest verifier sees a block, all other honest verifiers will only see that block. Thus, the following holds:

Observation. In Vault, a stamping certificate with one honest signature is valid.

Now let $T_{\text {stamping }}$ be the threshold of signatures needed to produce a valid stamping certificate, and let $\tau_{\text {stamping }}$ be the number of committee members elected in expectation

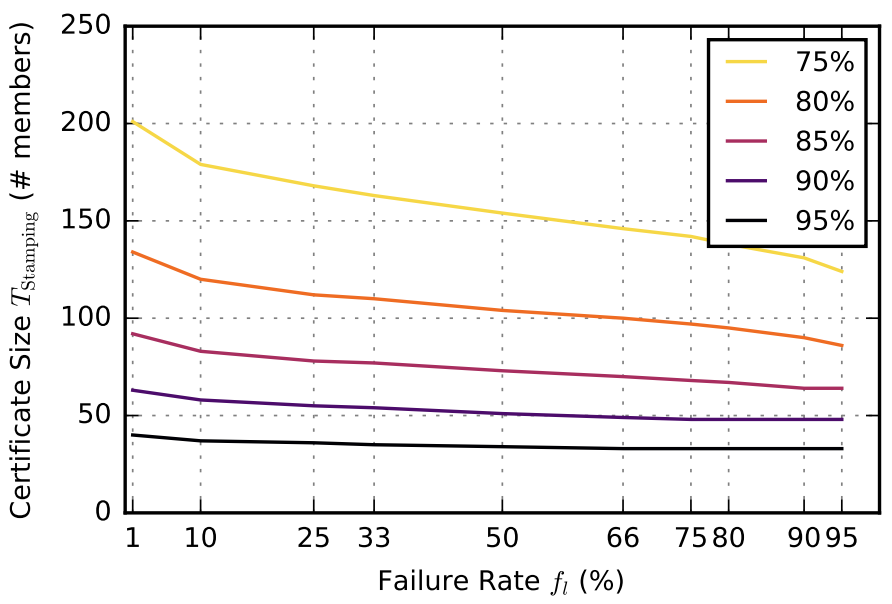

Fig. 9. Certificate sizes required to guarantee various liveness failure rates for a fixed safety failure rate of $2^{-100}$, given assumptions on the amount of honest stake in the system. Honesty assumptions have a significant effect on certificate size, which in turn influences the liveness failure rate.

to produce this certificate. Moreover, let $\gamma$ and $\beta$ be the actual number of honest and malicious users elected to some committee. We obtain two desirable properties, as follows:

Corollary. For Vault to produce certificates with a safety failure rate of $f_{s}$ and liveness failure rate of $f_{l}$, we must set $\tau_{\text {stamping }}$ as follows:

$$
\begin{aligned}
& \operatorname{Pr}\left[\gamma<T_{\text {stamping }}\right] \leq f_{l} \\
& \operatorname{Pr}\left[\beta \geq T_{\text {stamping }}\right] \leq f_{s}
\end{aligned}
$$

For simplicity, suppose that there are an arbitrarily large number of currency units in the system, and let each user in the system own one unit of currency. If $h$ is the proportion of honest users in the system and $\tau$ is the expected number of selected users following a cryptographic sortition, we have that the chance of sampling exactly $k$ honest users is

$$
\operatorname{Pr}[\gamma=k]=\frac{(h \tau)^{k}}{k !} e^{h \tau}
$$

while the chance of sampling exactly $k$ malicious users is

$$
\operatorname{Pr}[\beta=k]=\frac{((1-h) \tau)^{k}}{k !} e^{(1-h) \tau} .
$$

which follows from the application of the binomial theorem.

From Equation 1 and Equation 2 it follows that the following conditions must both hold:

$$
\begin{array}{cc}
\sum_{k=0}^{T_{\text {stamping }}-1} \frac{\left(h \tau_{\text {stamping }}\right)^{k}}{k !} e^{h \tau_{\text {stamping }}} & \leq f_{l} \\
\sum_{k=T_{\text {stamping }}}^{\infty} \frac{\left((1-h) \tau_{\text {stamping }}\right)^{k}}{k !} e^{(1-h) \tau_{\text {stamping }}} & \leq f_{s}
\end{array}
$$

Then it is evident that $\tau_{\text {stamping }}=120, T_{\text {stamping }}=100$ satisfy these conditions with $h=0.8, f_{s}=2^{-100}, f_{l}=0.65$.

Figure 9 illustrates the effects of changing $f_{l}$ for various values of $h$, fixing $f_{s}=2^{-100}$. 